\title{
Cambios en la comprensión del propio rol de extensionista a partir de la experiencia: un estudio latinoamericano
}

\author{
Changes in the understanding of the own role as extension worker as a \\ result of experience: a Latin American study
}

Fernando Pablo Landini ${ }^{1}$ (1)

${ }^{1}$ Consejo Nacional de Investigaciones Científicas y Técnicas (CONICET) e Universidad de la Cuenca del Plata: Posadas, Misiones, Argentina. E-mail: landini_fer@hotmail.com

Cómo citar: Landini, F. P. (2021). Cambios en la comprensión del propio rol de extensionista a partir de la experiencia: un estudio latinoamericano. Revista de Economia e Sociologia Rural, 59(2), e224267. https://doi.org/10.1590/18069479.2021 .224267

Resumen: La extensión rural constituye una práctica altamente compleja. En general, la bibliografía académica ha estudiado los cambios históricos que han acontecidos en los enfoques de extensión. Sin embargo, poca atención ha recibido el estudio de los cambios en los enfoques de los extensionistas a lo largo de su trayectoria profesional. En esta investigación se describe de qué manera se transforma el modo en que los extensionistas comprenden su rol a partir de la práctica y la experiencia. Se entrevistó a 133 extensionistas de seis países latinoamericanos. Las entrevistas fueron transcriptas y sometidas a un análisis de contenido. Los resultados muestran que los extensionistas, como resultado de la práctica y la experiencia, tienden a (1) ampliar y complejizar su forma de comprender la tarea de extensión, (2) tomar conciencia de las limitaciones de sus propios conocimientos y propuestas, (3) adoptar enfoques más dialógicos y constructivistas, y a (4) ser progresivamente más realistas. En paralelo, se observa que los enfoques de extensión se articulan fuertemente con las identidades y con las emociones de los extensionistas, lo que explica que impulsar cambios en el modo de pensar la extensión sea una tarea altamente compleja.

Palabras clave: extensión rural, asesoramiento técnico, cambios, identidad, emociones.

\begin{abstract}
Rural extension is a highly complex practice. In general, the academic literature has studied the historical changes that have taken place in the extension approaches. However, little attention has been given to the study of changes in the extension workers' approaches during their career path. In this study, it aims to describe how extension workers change their understanding of their role as a result of practice and experience. A total of 133 extension workers from six Latin American countries were interviewed. The interviews were transcribed and their content analyzed. The results show that extension workers, as a result of practice and experience, tend to (1) broaden and make more complex their understanding of extension work, (2) become aware of the limitations of their own knowledge and proposals, (3) adopt more dialogical and constructivist approaches, and (4) become more realistic. Besides, the research also shows that extension approaches are highly intertwined with the identities and the emotions of the extension workers, which explains why contributing to changes in how they understand their role is so difficult.
\end{abstract}

Keywords: rural extension, advisory service, changes, identity, emotions.

\section{INTRODUCCIÓN}

En América Latina, la mayor parte de los extensionistas rurales y asesores técnicos provienen de las ciencias agropecuarias (Landini \& Bianqui, 2014). Al iniciar su práctica profesional, estos se van a enfrentar con múltiples desafíos, dentro de los cuales se destaca la falta de práctica, a causa de una formación universitaria predominantemente teórica 
(Movahedi \& Nagel, 2012; Mojarradi \& Karamidehkordi, 2016). No obstante, resulta claro que la extensión rural y el asesoramiento técnico constituyen una práctica altamente compleja (Sulaiman \& Davis, 2012) que excede cualquier formación profesional específica. Esto se debe a que los productores no sólo requieren apoyo productivo, sino también comercial y organizacional (Landini, 2013), y a que los conocimientos temáticos específicos de los asesores y extensionistas necesitan complementarse con capacidades pedagógicas (Zuin et al., 2011) y de relacionamiento interpersonal (Landini, 2016a). En cierto sentido, se esperaría que asignaturas con contenidos sobre extensión y sociología rural impartidas en universidades y otros centros de estudio ofrecieran herramientas suficientes para abordar la complejidad de la práctica del extensionista. No obstante, se reconoce que materias puntuales resultan insuficientes para producir un impacto significativo en el perfil de los egresados en ciencias agropecuarias (Da Ros, 2012).

Al momento de enfrentarse a los espacios de práctica, extensionistas y asesores ponen en juego un conjunto de supuestos sobre cómo está organizada la realidad en su ámbito de intervención y cómo hay que actuar en ella para alcanzar resultados deseados (Houchens et al., 2012). Estos supuestos incluyen desde conocimientos técnicos sobre los cultivos y sus características, hasta cómo debe interactuarse con los productores al momento de dar una recomendación para generar cambios beneficiosos en sus modos de producir. Cuando las acciones que se implementan no llevan a los resultados deseados (lo que resulta frecuente en los momentos iniciales de práctica), se activarán diferentes procesos de aprendizaje, tanto de adquisición de nuevos conocimientos como de reorganización y ajuste de los supuestos previos que guían la práctica (Landini et al., 2017). Así, estas experiencias generarán cambios tanto en los conocimientos como en las identidades de los extensionistas (Cerf et al., 2011; Nettle et al., 2018), poniéndose en cuestión tanto los enfoques de extensión utilizados como la forma de comprender el propio rol de extensionistas o asesores.

En la literatura académica existe una cantidad considerable de trabajos que abordan los cambios históricos acontecidos en los enfoques de extensión rural. En general, estos estudios señalan el pasaje de modelos lineales de difusión tecnológica a propuestas que reconocen la existencia y el valor de múltiples tipos de conocimiento en el marco de procesos de comunicación horizontal y aprendizaje social (Machado et al., 2006; Klerkx et al., 2012). En esta línea, diferentes trabajos destacan la persistencia de modelos y prácticas tradicionales de extensión rural (Moschitz et al., 2015; Shukri Al-Rimawi et al., 2017) tanto a causa de trabas de nivel institucional como de la necesidad de desarrollar las capacidades de los extensionistas para actuar en el marco de los nuevos enfoques (Minh et al., 2010; Chowdhury et al., 2014).

Sin embargo, hoy no existe en la bibliografía científica ningún trabajo sistemático que estudie y describa de qué manera se va transformando a partir de la experiencia personal el modo en que los extensionistas rurales comprenden el trabajo de extensión y su propio rol como extensionistas o asesores, vacío que se propone abordar este artículo. Este trabajo parte del supuesto de que extensionistas rurales y asesores técnicos suelen enfrentar un conjunto de experiencias y desafíos similares en su trayectoria profesional, lo que llevaría a que exista un conjunto de transformaciones y cambios típicos o relativamente frecuentes en su identidad como extensionistas y en el modo de comprender su propio rol como resultado de la práctica y la experiencia. Claro está, esto no implica asumir que dichos cambios sean generalizables a todos los extensionistas y asesores, sino simplemente que existen ciertos cambios que son típicos o frecuentes y que pueden ser identificados y descriptos. Así, este artículo se propone como objetivo analizar y describir los cambios en los modos en que extensionistas rurales y asesores técnicos comprenden el trabajo de extensión y su propio rol como resultado de la práctica y la experiencia.

A primera vista, identificar y describir estos cambios puede parecer trivial. Sin embargo, un análisis más destallado pone en evidencia su valor. En efecto, si estos cambios en la comprensión del propio rol e incluso en la propia identidad como extensionistas constituyen el resultado de procesos de aprendizaje, comprender estos cambios permite identificar y sistematizar aprendizajes que suelen generarse a lo largo del tiempo. Ahora bien, al identificarlos y sistematizarlos, estos se convierten en herramientas para aportar a la 
formación en extensión rural, sea de futuros extensionistas como de quienes ya se encuentran cumpliendo el rol. En particular, estos resultados pueden ser utilizados como punto de partida para reflexionar críticamente sobre el rol de extensionistas y asesores y sobre los supuestos fundamentales del trabajo de extensión rural en el marco de procesos de formación de profesionales.

Ahora bien, reconociendo la posible existencia de particularidades asociadas a diferentes países, en este artículo se presentan los resultados de una investigación cualitativa realizada Argentina, Chile, Cuba, Ecuador, Guatemala y Uruguay, en la que se entrevistó a un total de 133 extensionistas rurales. Se destaca el interés de incorporar diferentes países y una muestra significativa de extensionistas a fin de identificar tendencias compartidas y diferencias entre ellos.

\section{EXTENSIÓN RURAL EN AMÉRICA LATINA: REFLEXIONES HISTÓRICAS}

La noción de extensión rural ha tenido importantes cambios a lo largo de la historia (Rivera, 2011), incluso al punto que algunos autores han propuesto abandonar el concepto (Leeuwis, 2004). Los actores que aquí denominamos 'extensionistas' han sido nombrados de múltiples maneras en América Latina según el contexto y la institución, incluyendo referencias como 'extensionistas rurales', 'agentes de extensión' 'asesores técnicos', 'profesionistas', 'agentes de desarrollo', 'técnicos de campo' y 'técnicos de ATER' (Asistencia Técnica y Extensión Rural), por algunas alternativas. Así, en este campo disputado es probable que ninguna definición específica permita recortar de manera definitiva al sujeto social al que se refiere. En cualquier caso, se puede definir al 'extensionista rural' como 'quien hace extensión rural', tomando de manera genérica la definición de Christoplos (2010) quien conceptualiza a la extensión rural como "las diferentes actividades de suministro de información y de asesoramiento pertinentes que solicitan los agricultores y otros actores en los sistemas agroalimentarios y el desarrollo rural" (p. 2).

La mayor parte de los autores acuerda que los sistemas de extensión rural latinoamericanos surgen a mediados del siglo XX con el apoyo activo de los Estados Unidos en la etapa posterior a la segunda guerra mundial (Otero \& Selis, 2016). En este marco, el modelo de extensión predominante seguía las premisas de la teoría de la modernización apoyada por la sociología estadounidense, que planteaba la necesidad de que los países 'pobres' o 'subdesarrollados' adoptaran las pautas y patrones culturales de las sociedades 'desarrolladas' (fundamentalmente los Estados Unidos). Así, cobra fuerza un modelo de extensión rural difusionista, caracterizado por la búsqueda de transferencia persuasiva de los conocimientos generados en las universidades y los centros de investigación a los productores, y por el rechazo de los conocimientos locales de los propios agricultores.

Durante las décadas del 60 y el 70, diferentes autores latinoamericanos ponen en cuestión las premisas de la teoría de la modernización, señalando que el 'subdesarrollo' no era consecuencia de las limitaciones culturales de los países 'periféricos' sino de la estructura desigual de las relaciones de poder a nivel internacional, propuesta que fue denominada teoría de la dependencia (Gumucio-Dagron, 2011). En este contexto se desarrollan formas de comunicación para el desarrollo de carácter alternativo, que reconocen el valor de las culturas locales y que ponen el énfasis en la implementación de dinámicas horizontales, críticas y participativas. Ubicado dentro de esta perspectiva, Freire (1973) propone una extensión rural entendida como proceso de comunicación horizontal centrado en el diálogo técnico-productor y el reconocimiento de los conocimientos y saberes de los agricultores. A partir de esto, puede pensarse la existencia de una tensión en América Latina entre enfoques de extensión rural difusionistas y enfoques dialógicos.

Hacia los años 80 y 90, asistimos a una crisis de la extensión rural en el marco de las políticas de ajuste estructural apoyadas en las premisas del neoliberalismo, lo que llevó a la desfinanciación, privatización y/o desaparición de gran parte de los sistemas de extensión rural latinoamericanos (Thornton, 2006; Diesel et al., 2008;). Con el nuevo siglo, y a partir de diferentes cambios políticos en América Latina, algunos autores han señalado el 'retorno de la extensión rural' a la agenda pública (Alemany \& Sevilla Guzmán, 2007; Aguirre, 2012). No obstante, visto en retrospectiva, se observa que este 'retorno' ha tenido múltiples 
altibajos y ha seguido diferentes trayectorias según los países, por lo que resulta difícil presentar descripciones de manera general.

En paralelo, también resulta conveniente señalar cambios más recientes acontecidos en los modos de abordar la extensión rural en América Latina, que involucran un pasaje de prácticas centradas exclusivamente en el vínculo extensionista-productor a propuestas que incorporan la coordinación entre diferentes actores para impulsar procesos de innovación y desarrollo. En este sentido, pueden observarse dos líneas complementarias, una centrada en los aportes del enfoque del desarrollo territorial (Torrado Porto \& Catullo, 2017), y otra que recupera una concepción de la innovación entendida como resultado de la articulación entre diferentes actores (Carrapizo et al., 2018). Así, el rol clásico del extensionista centrado en sus conocimientos sobre producción agropecuaria tiende a desdibujarse, acercándose cada vez más a la idea de agente de desarrollo rural.

En breve, a partir del recorrido realizado, se observa en América Latina un contraste entre enfoques difusionistas y dialógicos de extensión rural, que recientemente se complejiza al incorporar propuestas que recuperan la dimensión territorial y de articulación de actores en los procesos de desarrollo e innovación. No obstante, debe reconocerse que estas conceptualizaciones corresponden a un análisis que prioriza la discusión sobre modelos histórico-institucionales de extensión. A continuación, se analizan los enfoques de extensión desde la perspectiva de los sujetos.

\section{ENFOQUES DE EXTENSIÓN Y SUS CAMBIOS DESDE LA PERSPECTIVA DE LOS SUJETOS}

Existen estudios que mencionan algunos de los supuestos básicos a partir de los cuales los extensionistas guían sus prácticas y definen su rol. Por ejemplo, Höckert \& Ljung (2013) señalan que en Suecia los asesores técnicos tradicionalmente han sido formados como 'expertos', lo que los pone en una posición jerárquica en el vínculo con los agricultores. En esta línea, Minh et al. (2010) también señalan que los extensionistas no están acostumbrados a adoptar un rol de facilitadores, donde su conocimiento pueda ser puesto en cuestión por los productores, algo necesario para trabajar desde una extensión dialógica. En contraste, Ingram (2008) argumenta que los ingenieros agrónomos que trabajan como asesores pueden tomar diferentes actitudes, que van desde posicionarse como expertos a actuar como facilitadores de procesos reflexivos. Por su parte, a partir de un estudio realizado en diferentes países latinoamericanos, Landini (2016b) ha señalado que los extensionistas rurales pueden adoptar diferentes roles, aunque lo más frecuente es que comprendan su rol a partir de los principios del difusionismo, lo que resulta consistente con la adopción de un posicionamiento experto. Por último, Salomonsson et al. (2009) destacan que los ingenieros agrónomos suelen poseer una orientación productiva convencional estructurada a partir de principios productivistas, lo que les dificulta pensar relaciones complejas y sistémicas en el marco del funcionamiento de los agroecosistemas.

También existen autores que han analizado de qué manera los extensionistas rurales cambian el modo en que comprenden su rol, fundamentalmente a partir de talleres o espacios formativos. Landini et al. (2013), a partir de un estudio cuantitativo realizado en Paraguay, han señalado que los extensionistas rurales que tienen más años de experiencia poseen una orientación menos difusionista, lo que sugiere que la práctica y la experiencia podrían reducir actitudes típicas de un posicionamiento experto. En un trabajo de sistematización de talleres para extensionistas realizado en Francia, Albaladejo et al. (2007) destacan cambios históricos en el rol de los participantes, ahora más orientados a la facilitación de aprendizajes y vínculos que a la transmisión de conocimientos técnicos. En este contexto, los autores llaman la atención sobre el impacto de estos cambios en las identidades profesionales y en el modo en que los extensionistas se relacionan con el conocimiento. Por su parte, Cerf et al. (2011) en Francia y Landini \& Brites (2018) en Argentina muestran que, cuando los extensionistas reflexionan críticamente sobre su práctica, tienden a poner en cuestión supuestos y creencias instituidas sobre su propio rol, lo que les permite transformar sus modelos profesionales y sus prácticas como extensionistas. 
A la vez, en un estudio sobre un proceso de capacitación para asesores privados que trabajan en el sector lechero en Australia, Nettle et al. (2018) notaron que la incorporación de nuevas prácticas profesionales tiende a generar incertidumbre. No obstante, también observaron que la posibilidad de explorar nuevos roles profesionales en un contexto de seguridad y la retroalimentación positiva por parte de productores y de pares, reducen la incertidumbre y contribuyen a la adopción de nuevos roles. En particular, estos autores destacan que los procesos de cambio resultan más difíciles para quienes trabajan de manera independiente o en organizaciones que solo cuentan con uno o dos asesores, ya que poseen menos espacios para dialogar con pares y recibir retroalimentación relativa a la incorporación de nuevos roles y prácticas.

En este marco, cabe señalar que la capacitación en nuevas áreas de práctica contribuye a la transformación tanto del modo de trabajo como de las identidades de los propios extensionistas (Nettle et al., 2018). No obstante, hay que tener en cuenta que no es posible generar cambios significativos en los roles de los extensionistas a partir de actividades 0 eventos únicos (Chowdhury et al., 2014), ya que esto requiere tomar conciencia de las limitaciones de los modelos actuales de práctica y generar cambios a nivel actitudinal, todo lo que toma tiempo (Minh et al., 2010). En este marco, cobra sentido el valor que diferentes autores dan a la capacidad de los extensionistas para reflexionar sobre su propia práctica (Tarekegne et al., 2017; Landini \& Brites, 2018), en tanto herramienta fundamental para adaptar e innovar en el modo de hacer extensión.

En resumen, la revisión de antecedentes muestra que, en general, los extensionistas rurales y los asesores técnicos tienden a adoptar una posición de expertos a partir de su formación universitaria y a posicionarse desde una perspectiva de extensión lineal o difusionista. En paralelo, se observa que los procesos de formación y de reflexión sobre la práctica juegan un rol de gran importancia en la transformación de los roles, las identidades y las prácticas de los propios extensionistas. En particular, se reconoce que se trata de procesos no lineales, que toman tiempo, y que se apoyan en el vínculo y la retroalimentación proveniente de pares y de productores.

\section{METODOLOGÍA}

El presente trabajo se enmarca en un estudio más amplio orientado a comprender de qué manera extensionistas rurales y asesores técnicos aprenden en diferentes contextos a llevar adelante su labor. En particular, en este artículo se analizan y describen los cambios en los modos en que extensionistas rurales y asesores técnicos comprenden el trabajo de extensión y su propio rol como resultado de la práctica y la experiencia.

El enfoque adoptado para la investigación fue cualitativo, descriptivo e interpretativo (Taylor \& Bogdan, 1990). Se realizaron un total de 86 entrevistas (68 individuales y 18 grupales) a extensionistas rurales y asesores que trabajan en diferentes instituciones en seis países latinoamericanos: Argentina, Chile, Cuba, Ecuador, Guatemala y Uruguay. Las entrevistas se realizaron en 2016 y 2017 en Argentina y en 2017 en el resto de los países. A nivel metodológico, siguiendo a Christoplos (2010), en este trabajo se utilizan los conceptos de extensionista rural y de asesor técnico de manera intercambiable como forma de referirse al conjunto de actividades orientadas a ofrecer información, apoyo y asesoramiento a agricultores y a otros actores de los sistemas agroalimentarios o vinculados con el desarrollo rural. En lo que resta del artículo, a fin de no resultar repetitivo, se utilizarán ambas expresiones de manera intercambiable, aunque priorizándose la referencia 'extensión rural'.

En total se entrevistó a 133 extensionistas. En cada país se realizaron 15 entrevistas (entre dos y cuatro grupales, el resto individuales), con la única excepción de Cuba, donde se realizaron 11 (ocho individuales y tres grupales). Los países fueron seleccionados por conveniencia, a partir del vínculo del autor con instituciones locales de extensión o con investigadores locales.

En los países incluidos en el estudio que cuentan con sistemas de extensión públicos de alcance nacional, se optó por entrevistar a quienes trabajan en los Ministerios de Agricultura o sus equivalentes (Argentina: Instituto Nacional de Tecnología Agropecuaria [INTA] y 
Subsecretaría de Agricultura Familiar [SsAF]; Chile: Programa de Desarrollo Local [PRODESAL] del Instituto de Desarrollo Agropecuario [INDAP]; Ecuador: Ministerio de Agricultura y Ganadería [MAGA]; Guatemala: Ministerio de Agricultura, Ganadería y Alimentación [MAGA]). En Cuba se entrevistó a extensionistas y asesores de productores que trabajan en diferentes instituciones, como ser municipios, institutos de investigación o unidades de producción de carácter estatal o cooperativo. En Uruguay se contactó a asesores independientes, a técnicos de la Cooperativa Agraria Nacional (COPAGRAN) y a extensionistas que trabajan en diferentes instituciones públicas vinculadas con la producción agropecuaria y el desarrollo rural.

Los territorios donde se tomaron las entrevistas en cada país fueron: provincia de Corrientes (Argentina), Región Metropolitana (Chile), provincia de Granma (Cuba), provincia de Cotopaxi (Ecuador), departamentos de Chiquimula y Zacapa (Guatemala) y departamentos de Paysandú y Salto (Uruguay). La edad media de los entrevistados fue de 42 años, el 35\% de los cuales fueron mujeres. La experiencia media como extensionistas varió entre 6 años (Ecuador) y 18 años (Cuba), con una media general de 12 años y medio. La mayoría posee título en el ámbito de las ciencias agropecuarias, con solo el 5\% de los entrevistados con título universitario en ciencias sociales.

Las entrevistas fueron semiestructuradas y se realizaron a partir de un protocolo común, que incluyó múltiples preguntas relativas al aprendizaje de los extensionistas rurales y a los procesos cognitivos y reflexivos relacionados. No obstante, este protocolo se utilizó de manera flexible teniendo en cuenta las respuestas de los entrevistados y los contextos institucionales y materiales de su práctica (Díaz-Bravo et al., 2013), algo particularmente importante teniendo en cuenta la diversidad de países e instituciones involucradas. Todas las entrevistas fueron grabadas y transcriptas de manera literal. Se obtuvo en todos los casos consentimiento informado para la grabación y para la participación en la investigación. La mayor parte de las entrevistas estuvieron a cargo del autor del artículo. En Chile y Ecuador se contó con el apoyo de investigadores locales que realizaron la mitad de las entrevistas correspondientes a ambos países. Todas las entrevistas realizadas en Cuba estuvieron a cargo de una investigadora del país.

El análisis fue realizado con el apoyo del software Atlas.Ti para análisis cualitativo. El primer paso consistió en la identificación de todos aquellos fragmentos de las entrevistas en los cuales se hacía referencia a cambios en los enfoques de extensión o en el rol del extensionista rural. A partir de esto, se realizó una lectura minuciosa de todos ellos, lo que permitió construir un listado de categorías para ordenar el material textual. Estas categorías corresponden a dos grandes grupos, uno relativo a las causas de los cambios en los enfoques y en la concepción del rol, y otro, el más significativo cuantitativamente, relativo a los cambios en sí mismos. En los siguientes apartados se presenta una descripción ordenada e integrada de estas diferentes categorías, a fin de evidenciar los cambios en los enfoques de extensión y en la percepción del propio rol como extensionistas de los entrevistados.

A los fines de presentar evidencia de los resultados obtenidos, se incluyen citas textuales. Para explicitar el origen de cada cita se utiliza el siguiente código luego de cada cita: $\mathrm{H}=$ hombre; $\mathrm{M}=$ mujer; Argentina $=\mathrm{AR}$; $\mathrm{Chile}=\mathrm{CH}$; $\mathrm{Cuba}=\mathrm{CU}$; Ecuador=EC; Guatemala=GT; Uruguay=UY. A esto se agrega un número, que corresponde al número del archivo de la entrevista en el Atlas.Ti. Así, por ejemplo, M.GT.50 significa que la entrevistada es una mujer extensionista de Guatemala, cuyo número interno de entrevista es el 50.

\section{RESULTADOS}

\subsection{Los cambios en los enfoques y en la comprensión el propio rol como constante}

La mayor parte de los extensionistas entrevistados reconocen o describen cambios en sus enfoques de extensión o en su modo de interpretar su rol en comparación con el momento en que se iniciaron en el trabajo. Solo siete indicaron no haber cambiado su enfoque o no haberlo hecho de manera significativa. Por ejemplo, cuando se preguntó a 
una entrevistada si había cambiado su forma de ver la extensión rural a lo largo del tiempo, ella respondió: "yo no, yo sigo igual, yo sigo porque a mí me encantan los animales" (M.CU.32). No obstante, aún estos casos de 'ausencia de cambio' resultan dudosos, al observarse que en tres de ellos se describen transformaciones en otros fragmentos de las entrevistas. En este sentido, un extensionista chileno, que había dicho que su enfoque de extensión "no cambió mucho, porque yo me crie con mi tío y mi abuelo que son agricultores" (H.CH.77), también señaló casi en el mismo momento que "yo antes estaba acostumbrado a [...] si no eran 20 hectáreas producidas no existía [como productor] [...]. Acá [...] me di cuenta que la gente [...] con un gallinero y un patio chiquitito que tenga 150 gallinas, la persona gana plata, ahí cambió mi forma de pensar" (H.CH.77). A partir de esto puede afirmarse que la amplia mayoría de los extensionistas modifica con el paso de los años sus enfoques y abordajes iniciales.

\subsection{Orígenes de los cambios}

A partir de las entrevistas se identificaron tres fuentes u orígenes de los cambios en los enfoques de extensión y en el modo en que los extensionistas comprenden su rol. En orden creciente de importancia según las entrevistas, estos son: transformaciones de los contextos, cambios en las instituciones y sus abordajes, y la experiencia y la práctica.

Respecto de los cambios contextuales, uno de los entrevistados destaca que el enfoque de extensión "va cambiando porque los productores van cambiando [...] cambia la forma en que ellos se plantan en una reunión, la forma en que ellos te plantean las demandas de la comunidad" (H.AR.1). Así, cambios en las demandas de los productores pueden impulsar cambios en el rol y el enfoque de los extensionistas. Otros señalaron la incidencia de cambios en la disponibilidad de recursos económicos u operativos (como combustible, por ejemplo) para llevar adelante el trabajo: "ese enfoque cambió por la necesidad de cambio. Porque los enfoques convencionales dejaron de funcionar. Sobre todo, cuando me tocó trabajar en el contexto [...] de carencia de insumos, de apoyo político y de todo tipo, de apoyo materias, de escasez de combustible" (H.CU.35). Finalmente, hay quienes señalaron el impacto de fenómenos climáticos, como ser períodos prolongados de sequía que requieren cambios en las estrategias de extensión.

Se observa entonces que cambios en el contexto inciden en los modos en que los extensionistas abordan su trabajo, aun cuando esto no signifique necesariamente cambios significativos o profundos en la comprensión de su rol. De hecho, queda claro que los cambios surgen a causa de restricciones o presiones del contexto, no a partir de cambios internos en los modos de comprender o conceptualizar la tarea que se lleva adelante. De todas formas, debe tenerse en cuenta que los nuevos contextos pueden impulsar procesos de aprendizaje y/o de reflexión que lleven a cambios internos duraderos en el modo de pensar el propio rol, en contraste con lo que serían cambios de enfoque circunstanciales sostenidos en restricciones externas. Esto puede apreciarse en la siguiente cita: "uno tiene que cambiar la forma de trabajar debido a los recursos con los que cuenta, a mí la falta de recursos me enseñó mucho a gestionar, a gestionar y a ser muy pidón" (M.GT.54). Como se observa, el nuevo enfoque orientado a la generación de alianzas para superar la falta de recursos surge como un aprendizaje, que seguramente persistirá en el repertorio de la entrevistada aun cuando las restricciones presupuestarias del contexto disminuyan.

Por su parte, otros entrevistados destacaron la incidencia de cambios en los marcos institucionales como origen de transformaciones significativas en su modo de trabajar como extensionistas. En general, estos cambios aparecen asociados a casos o situaciones concretas donde se redefinen los enfoques institucionales (cambios paradigmáticos) o a reestructuraciones en los proyectos o programas en los que trabajan los entrevistados. En la investigación se identificaron cuatro ejemplos (en igual número de países) de cambios significativos en el modo de hacer extensión a partir de cambios institucionales.

Un entrevistado del Instituto Plan Agropecuario de Uruguay señala: "antes del [año] 2000 nosotros trabajábamos como una institución que hacía transferencia tecnológica, y del 2000 para acá hicimos un cambio relativamente importante, que todavía lo estamos 
procesando, en el cual se trabaja sobre la consigna del aprendizaje conjunto con los productores" (H.UY.72). Luego agrega: "para nosotros fue un cambio de paradigma relativamente brusco, en el cual hubo que cambiar el chip que teníamos puesto todos en la cabeza, y eso llevó un proceso de años, [...] podría decir que llevó 15 años" (H.UY.72).

Como se observa en el resto de los casos (con excepción del ecuatoriano donde el foco está en cambios en la estructura de los proyectos relacionados con una disminución en la entrega de insumos), se trata de transformaciones profundas de enfoque institucional que llevan tiempo de consolidación y que requieren cambios concomitantes en el modo de pensar el rol y la tarea de extensión por parte de quienes se desempeñan como extensionistas. Esto hace que las instituciones propongan estos cambios en paralelo con procesos de capacitación y de reflexión en relación a los nuevos enfoques: "cuando se incorporó esta nueva visión [...] fuimos adquiriendo nuevos conocimientos y herramientas a través de la capacitación" (H.AR.1), "empezó un proceso en nuestro instituto [...] de reflexión sobre lo que es la extensión [...] y así caemos en el paradigma participativo" (H.CU.35).

En resumen, se observa que los cambios institucionales en los enfoques de extensión pueden operar simplemente a partir de incidir en los recursos que se asignan circunstancialmente a diferentes proyectos y programas. No obstante, la mayoría de los casos se relacionan con cambios en los paradigmas de extensión que utilizan las instituciones, lo cual requiere de transformaciones profundas en las creencias y visiones de los propios extensionistas. En este estudio, dichos cambios han sido impulsados a partir de procesos de capacitación y reflexión.

A fin de comprender mejor cómo inciden los cambios de enfoque institucional en los enfoques y prácticas de los extensionistas, resulta interesante analizar qué fue lo que impulsó dichos cambios en las instituciones. En primer lugar, las entrevistas muestran el rol clave que pueden jugar los propios actores de las instituciones y sus autoridades en el impulso de nuevos enfoques de extensión: "estuve a cargo de la coordinación [...] fuimos avanzando en cambiar un poco el enfoque" (H.AR.1), "empezó un proceso en nuestro instituto [...] de reflexión sobre lo que es la extensión [...], y así caemos en el paradigma participativo" (H.CU.35). Así, se observa la capacidad de agencia de los propios integrantes de las instituciones como fuente de las transformaciones en los enfoques institucionales de extensión.

Ahora bien, pese a lo anterior, también es necesario reconocer que estas transformaciones se dieron en una línea específica, consistente con los cambios en los modelos conceptuales utilizados para pensar la extensión y el desarrollo rural a nivel internacional, lo que hace pensar en la incidencia de dichos modelos en los enfoques institucionales. Así, los cambios descriptos en la cita Argentina se orientan hacia el desarrollo territorial, en Cuba se menciona una reorientación hacia metodologías participativas y en Uruguay se plantea un alejamiento del paradigma de transferencia tecnológica. Por último, si bien no fue explicitado en las entrevistas, también debe considerarse la incidencia de cambios a nivel de autoridades nacionales de cada país, las cuales definen las orientaciones generales que van a dar forma a las políticas públicas de extensión, marcadas según diferentes ideologías.

Ahora bien, más allá de la incidencia de los cambios en los contextos y en los enfoques institucionales, hay que tener presente que los extensionistas señalan a la experiencia y la práctica como origen principal de transformaciones en sus enfoques de trabajo y en la comprensión de su rol. La siguiente cita sirve de ejemplo: "al principio [...] uno desconoce muchas cosas [...] uno como que va madurando más sus conocimientos [...] ya el enfoque de uno va cambiando. Al inicio uno quizás lleva los principios básicos de la universidad, y muchas veces no están acorde a la realidad de las comunidades rurales" (H.GT.51). En el siguiente fragmento el proceso de repensar el rol se observa de manera más impactante: "Ideológicamente fue cambiando [...] para mí fue muy fuerte. Las organizaciones me enseñaron a ser menos soberbia, desde el saber profesional" (M.AR.11). Como en muchas otras citas, aquí se observa un proceso de reelaboración y ajuste del modo de enfocar el trabajo, a partir de poner en cuestión supuestos y creencias previas, lo que lleva a nuevos abordajes y a nuevas formas de plantearse el rol y la relación con los productores. 


\subsection{Cambios en los enfoques de extensión y en la comprensión del rol del extensionista basados en la práctica y la experiencia}

Existen diferentes cambios en los enfoques de extensión y en la forma de comprender el rol del extensionista que se identificaron en esta investigación como consecuencia de la práctica y la experiencia. En cualquier caso, debe tenerse presente que la experiencia no es ajena a los cambios en el contexto y en las instituciones, ya que estos últimos son los que dan forma a los escenarios donde se llevan adelante las prácticas. A continuación, se describen los cambios más significativos.

\subsubsection{Ampliación y complejización del campo de acción}

En numerosas entrevistas los extensionistas destacaron que cuando finalizaron su formación de grado tenían una mirada exclusivamente técnico-productiva de su labor: "cuando egresamos [nuestra misión] era [...] planificar la cuestión productiva, abstraído de lo social [...] 'yo tengo que producir más"' (H.AR.14). Sin embargo, en 17 entrevistas (fundamentalmente de Argentina y Uruguay) se describieron procesos que llevaron a ampliar la visión tanto de las áreas de acción del extensionista como de las variables a tener en cuenta para una labor exitosa. En este sentido, diferentes entrevistados señalaron la necesidad de que la extensión preste atención no solo a lo productivo sino también a problemáticas asociadas, como ser la comercialización y la industrialización: "[hay que] tratar de cerrar toda la cadena, que es la comercialización, la industrialización" (M.EC.19).

En paralelo, numerosos entrevistados señalaron que el trabajo de extensión incluye múltiples dimensiones de índole social. Así, por un lado, se reconoce que la extensión se apoya en procesos de educación y formación que exceden lo propiamente productivo, por lo que se necesitan extensionistas con capacidad pedagógica. Al mismo tiempo, la experiencia hace evidente que un buen trabajo de extensión necesita apoyarse en la construcción de un buen vínculo con los productores, a partir sentarse, escucharlos y conversar con ellos: "primero hablar con los productores, conocerlos, saber un poco de ellos" (M.UY.62).

En la misma línea, diferentes entrevistados destacaron que, para hacer un buen diagnóstico de una situación o problemática, así como para dar recomendaciones apropiadas, hay que tener en cuenta a la familia, sus características y sus dinámicas: "me he volcado hacia el diagnóstico a nivel integral, sistémico, de toda la realidad de una familia, y la mirada es hacia la familia, varón, mujer, mujer, varón" (H.UY.63). En definitiva, hay una percepción de que el trabajo de extensión posee un fuerte componente social que involucra desde el relacionamiento interpersonal y las dinámicas familiares hasta las condiciones de vida de las personas. Claro está, esto no significa que se plantee que el trabajo de extensión y de asesoramiento técnico tenga que plantearse siempre en estos términos, pero sí que en gran parte de las situaciones el no hacerlo va a llevar a dar respuestas inadecuadas a los productores.

\subsubsection{Pérdida de la soberbia intelectual y reconocimiento de las limitaciones del conocimiento propio}

Numerosos extensionistas comentaron que la experiencia les permitió tomar conciencia de las limitaciones de sus propios conocimientos y propuestas técnicas (en 11 entrevistas), y que con el tiempo fueron reconocimiento progresivamente el valor de los saberes de los productores (12 entrevistas) (mayormente de Argentina, Guatemala y Uruguay). En este sentido, algunos señalaron que: "uno cree que lleva toda la tecnología y lleva toda la solución a los problemas [sin embargo] cuando se da cuenta en el campo, no es tan fácil llegar e implementar lo que uno lleva" (H.GT.59). Esto lo aclara otro extensionista al argumentar que: "al inicio uno quizá lleva ya los principios básicos de la universidad, y muchas veces no están acorde a la realidad de las comunidades rurales" (H.GT.51). Así, numerosos entrevistados reconocen que el conocimiento aprendido en las universidades e institutos de formación posee limitaciones para ser aplicado de manera directa en los contextos de práctica en los que se desenvuelven. Más aún, admiten que sus 
propios conocimientos son limitados: "desde el inicio pensamos que lo sabíamos todo, que nos enseñaron en la universidad, y cuando llegamos al campo nos dimos cuenta que faltó muchos conocimientos" (M.EC.28).

En paralelo al reconocimiento de la limitación de los conocimientos propios, se observa una progresiva valorización de los saberes de los productores. Uno de los entrevistados señala: "Ios productores tienen muchísimo conocimiento desarrollado sobre la base de su experiencia, en el campo, en el trabajo" (H.UY.72). Por esta razón, ellos proponen que: "hay que aprender más de la gente" (H.UY.61). Ante esta situación, uno podría asumir que esta conciencia no es algo que se genera como parte de la experiencia, sino que es algo que forma parte del bagaje previo de quienes empiezan a trabajar como extensionistas. Sin embargo, cuando se preguntó esto las respuestas fueron claras, explicándose que uno realmente toma conciencia del valor del conocimiento de los productores con el tiempo: "llegué [a esta conclusión] en el transcurso del tiempo, el momento exacto no sabría" (H.AR.9).

En 11 entrevistas, distribuidas en los distintos países, el reconocimiento de las limitaciones de los propios conocimientos, sumado a la valorización de los saberes de los otros, llevó a describir un cambio a nivel actitudinal en el vínculo con los productores. Se trata del pasaje de una posición intelectual soberbia en la cual se desconocían las limitaciones de los propios conocimientos y se negaba el valor del conocimiento empírico de los productores, a una actitud personal mucho más humilde y realista. Una de las entrevistadas explica: "es que uno llega... como de la universidad... como que uno se las sabe todas [...] entonces como que uno se hace más humilde" (M.CH.78). En la misma línea, otro extensionista señala: "uno tiene que ser un amigo más del productor, no el ingeniero, 'yo soy el más...' [...] son las cosas que he aprendido [...] lo tan humilde que yo puedo ser con ellos" (H.EC.16).

\subsubsection{Reconocimiento del productor como sujeto con independencia y autodeterminación}

En un total de 27 entrevistas (la mayoría de ellas de Argentina y Uruguay), los entrevistados explicaron haber aprendido (o tomado verdadera conciencia) de que los productores poseen una forma de pensar, una lógica y unos objetivos propios que no se identifican con la lógica a partir de la cual piensan los extensionistas. De esta forma, se reconoce al productor como sujeto con independencia y autodeterminación, lo que lleva a ubicarlo en un rol estructurante y en el lugar central del proceso de extensión. Así, la extensión deja de tener que ver primariamente con la producción para tener que ver con el productor, sus intereses y sus decisiones.

En primer lugar, numerosos entrevistados destacaron que el extensionista puede dar recomendaciones y hacer diferentes propuestas a los productores, pero que, si ellos no acuerdan o no están interesados con lo que se les propone, entonces la iniciativa no va a salir adelante. Uno de los entrevistados explica: "podés darle todas las propagandas que quieras, o podés hacer todo lo que quieras [pero] si la otra parte no está de acuerdo, eso no tiene vida" (H.AR.3).

En esta línea, diferentes extensionistas agregan que los productores escuchan, pero sin tomar como 'palabra santa' lo que se les dice, y que si por alguna razón es posible llevar adelante una iniciativa con la que los productores no acuerdan (por ejemplo, como obligación para recibir algún subsidio), lo más probable es que ésta termine fracasando.

Los entrevistados identificaron dos factores que les ayudan a explicar por qué los productores muchas veces toman decisiones por fuera de lo que parece lógico al extensionista. Por un lado, reconocen que los conocimientos, las experiencias y la cultura de los productores constituyen una fuente de sabiduría que los guía en su práctica. Y por el otro, destacan que ellos tienen razones, objetivos y puntos de vista propios, que no se identifican con el modo de pensar del técnico, y que deben ser comprendidos y valorados. Esto puede observarse en la siguiente cita: "el otro [el productor] también tiene un montón de saberes, tiene su vida y circunstancias y problemáticas, y uno [tiene que] ubicarse en su lugar" (H.AR.9). De esta forma, la comunicación interpersonal adquiere un rol fundamental: 
"esa misma comunicación es la que a nosotros nos sirve como para entender los conocimientos de ellos y que ellos también nos puedan entender a nosotros" (H.CH.76).

Es a partir de esto que uno de los entrevistados describe al trabajo de extensión rural como "una interrelación humana" (H.UY.63). En este marco, numerosos extensionistas plantean que el escuchar a los productores y el establecer una relación de confianza con ellos constituye un componente central de la tarea de extensión, algo que llamativamente no formaba parte de sus expectativas y objetivos iniciales como extensionistas: "hay un tema humano de por medio. Entonces, de partida aprendes a relacionarte con 180 personalidades diferentes. [...] acá uno tiene que usar una psicología increíble. Y lo primero que tienes que hacer, antes de pensar en dar ninguna indicación, es ganarte la confianza del agricultor" (M.CH.89). De esta forma, la capacidad de escuchar y de generar relaciones interpersonales de confianza se convierte en un valor, al igual que desarrollar una forma de hablar o un vocabulario acorde con la forma de hablar de los productores: "al principio cuando yo llegué al trabajo, no le hablaba simple a las personas, [...] ahí yo cambié mi chip, ahí me puse menos universitaria y me puse más acorde, más simple" (M.CH.85).

\subsubsection{De la transferencia de recetas a la negociación y a la construcción de acuerdos}

En el subtítulo previo se afirmó que un cambio central en el modo de pensar el trabajo de extensión de los entrevistados se relaciona con el reconocimiento del rol central que juegan los objetivos, las prioridades y la toma de decisiones de los productores en el modo en que éstos responden a las recomendaciones y propuestas de los extensionistas. A partir de esto, en 16 entrevistas (también mayoritariamente de Argentina y Uruguay) se describió el pasaje de un abordaje centrado en decirles a los productos qué es lo que tienen que hacer (a partir de transferirles recomendaciones y prácticas 'correctas') a uno caracterizado por el diálogo y la construcción de acuerdos desde una perspectiva horizontal.

En primer lugar, diversos entrevistados señalaron que su intención inicial (relacionada con el momento de 'salir de la universidad') era simplemente decirles a los productores lo que tenían que hacer. Un extensionista lo comenta: “antes era mucho más lineal, por lo menos cuando salimos de la facultad, era 'tenemos que ir con los productores, decirle cuánto tienen que aplicar, cómo se cura, cómo se hace esto, lo otro"' (H.AR.10). Otro entrevistado lo explica a nivel conceptual "uno queda anclado en los métodos tradicionales del extensionismo [...] ese modelo lineal lo traemos nosotros muy fuertemente arraigado. El cambio de actitud es asumir [...] que el proceso de extensión debe ser más horizontal" (H.CU.35). A partir de su experiencia, diferentes entrevistados concluyen que el extensionista no debe buscar transferir o imponer su punto de vista a los productores: "tampoco puedes llegar a un grupo de personas y como obligar que apliquen" (H.EC.28), "no lográs nada" (H.AR.4), "no [hay que] imponer cosas" (H.AR.10).

A partir de esto, múltiples entrevistados proponen una estrategia de trabajo distinta, que contrasta con sus impulsos iniciales y que surge de su experiencia. Así, en primer lugar, destacan la necesidad de tomar como base los intereses y preferencias de los productores para, a partir de ellos, poder hacer propuestas y recomendaciones que las tengan en cuenta. Una extensionista describe este cambio desde su experiencia: "creo que el gran cambio [...] [fue poner] primero el factor humano, primero hablar con los productores, conocerlos, saber un poco de ellos, qué es lo que quieren, cuáles son sus objetivos y en base a eso trabajar" (M.UY.62).

A la vez, por otro lado, diferentes entrevistados también destacan que es necesario negociar y generar acuerdos con los productores, incluyendo las experiencias, conocimientos y puntos de vista de ambos: "podemos empezar a compartir puntos de vista y modificar algo, pero no es todo como yo digo" (H.AR.5). Incluso, es a partir de este diálogo técnico-productor que se generan espacios para que los productores puedan pensar o readaptar propuestas técnicas, haciéndolas más acordes a su realidad y contexto, lo que aumenta su aplicabilidad.

En cualquier caso, debe tenerse presente que no todos los entrevistados comparten la misma forma de pensar, y en diferentes oportunidades se observa la persistencia de enfoques de extensión rural que ponen el énfasis en la persuasión de los productores para 
la adopción tecnológica, particularmente en Cuba y Ecuador. Así, por ejemplo, un profesional ecuatoriano plantea que "hay que ganarse al productor, tú vienes con un pensamiento diferente y [hay que] tratar de cambiarlos a ellos en sus formas de cultivar, porque a veces son tradicionalistas" (M.EC.30). De la misma forma, un extensionista cubano argumenta que "es un trabajo bastante complejo [...] porque todo el mundo piensa diferente [...] y todos no tienen el mismo pensamiento, el mismo nivel cultural, y entonces hay que convencerlos" (H.CU.39).

En resumen, los datos muestran que la amplia mayoría de los extensionistas parte de un enfoque de extensión cercano al difusionismo cuando se inician. A partir de allí, un porcentaje significativo lo va transformando progresivamente en línea con una propuesta más horizontal y dialógica a partir del aprendizaje derivado de su experiencia, aun cuando esto no suceda en todos los casos.

\subsubsection{Realismo y desidealización}

En 12 entrevistas (fundamentalmente de Argentina y Uruguay) los extensionistas señalaron que la experiencia les permitió ser más realistas en relación al contenido y a lo que puede ser alcanzado por medio del trabajo de extensión. Incluso, en algunos casos puede observarse cierta desidealización, tanto de los productores como del propio trabajo.

Varios entrevistados destacaron que los cambios que se pueden generar a partir del trabajo de extensión son más lentos de que ellos pensaban al inicio. Un entrevistado lo explica diciendo: "vos ves que los cambios se van dando mucho más lento de lo que uno por ahí cree que se puedan dar" (H.AR.4), por lo que "hay que ir más despacio" (H.UY.73). En paralelo, otros entrevistados comparten su experiencia de que los cambios que se generan con el trabajo de extensión no son tan importantes o significativos como lo imaginaban en un principio: "cuando me recibí salíamos a hacer la revolución y a cambiar el mundo, y ahora, si logro cambiar la realidad de un tipo, quedo contentísimo" (H.UY.67).

Este realismo, derivado de la experiencia, también alcanza a la situación productiva de los agricultores, en particular a la dotación de capital y a los recursos disponibles. Si bien esta idea no es tan directa como las anteriores, hace referencia a la toma de conciencia de que los productores no cuentan con recursos para implementar gran parte de los esquemas productivos aprendidos en las universidades o considerados 'de punta'. Esto podría expresarse diciendo que la experiencia permite tomar conciencia de que las alternativas productivas aprendidas no son viables para la mayoría de los productores. Y esto, incluso, "aun en productores grandes" (H.UY.73). Un técnico guatemalteco lo plantea desde su experiencia: "estaba acostumbrado a producir en cantidades, con tecnologías avanzadas como fertilizantes y productos innovadores, ahora [que soy extensionista] tengo que aplicar tecnología en la producción con materiales que se encuentran en el área para poder producir" (H.GT.55).

Finalmente, este realismo también incluye un proceso de desidealización de los productores y de la comunidad, que puede tener diferentes dimensiones. En este sentido, en las entrevistas se comentan experiencias en las que se tomó conciencia de que los productores no siempre son solidarios ni se comprometen con sus pares en el marco de cooperativas o procesos comunitarios, que suelen armar un discurso para decirle a las instituciones lo que éstas quieren oír para conseguir recursos, o incluso que no siempre se comprometen con iniciativas o procesos que los pueden beneficiar. Como ejemplo puede citarse el caso de un extensionista que, no sin cierto pesar, dice que hay que "ser más selectivo por ejemplo con la gente [que se incorpora a un proyecto], porque hay gente que no tiene ganas de hacer [es decir, trabajar o comprometerse]" (H.AR.3). En la misma línea, otro entrevistado plantea "tú llegas con harta [mucha] ilusión a esta pega [trabajo] [...] pero al final muchas veces la gente le desilusiona, [...] te esforzaste, y la tiraste para arriba, y después te pega la media desconocida" H.CH.90. 


\subsubsection{Trabajo de extensión y su impacto emocional}

En 18 entrevistas (fundamentalmente de Guatemala, Chile, Ecuador y Uruguay), los participantes hicieron referencia a emociones y sentimientos personales vinculados con el trabajo de extensión y la experiencia de ser extensionistas. Si bien el impacto emocional del trabajo de extensión no puede ser considerado en sí como un cambio en el enfoque de trabajo o en el modo de comprender el propio rol, en este estudio fue posible percibir cambios derivados de dichos impactos emocionales.

En el marco de las entrevistas se pudieron identificar tres situaciones que suelen impactar (muchas veces de manera profunda) en las emociones de los extensionistas. En primer lugar, diferentes entrevistados explicaron que su trabajo los llevó a conocer a productores que se encuentran en condiciones de vida difíciles, que deben hacer grandes esfuerzos para vivir, incluyendo situaciones graves de pobreza. En este contexto, algunos comentan que esto los llevó a tener conciencia de una realidad que no tenían presente o que les resultaba ajena, a valorar más y a sentirse agradecidos por las cosas que tenían, o incluso a sentir angustia o tristeza por la situación de necesidad y vulnerabilidad de las personas con las que trabajaban. Esto puede apreciarse en las palabras de uno de los entrevistados: "la gente de la montaña llega a trabajar conmigo y uno no sabía ni cómo viven [...] en cambio uno ya que sube ahí, que mira cómo son sus condiciones y todo, ya uno los comprende más [...] llego a unas comunidades que me vengo triste por cómo están" (H.GT.56).

Otro de los sentimientos generados en el vínculo con los productores es el enojo o el sentirse defraudados, a causa de diferentes situaciones, fundamentalmente desconocer los esfuerzos hechos por los extensionistas para apoyarlos o no aprovechar (por aparente negligencia o mala voluntad) oportunidades que se les ofrecen. Una extensionista comenta: "en otra etapa uno quería estar [...] como liderando el proceso [en el trabajo con grupos o asociaciones] [...], y uno se re calentaba con los conflictos, te agarrabas desde preocupación hasta amargura [...] y te quemabas mucho" (M.UY.74). Al mismo tiempo, algunos entrevistados también comentan que trabajando se generan fuertes vínculos afectivos con los productores, que llevan a la construcción de relaciones de compañerismo y amistad: "se va formando amistades, y prácticamente nuestros productores forman parte ya de nuestra familia" (M.EC.28).

De esta manera, se observa que el trabajo de extensión compromete emocionalmente al propio extensionista, al facilitar el surgimiento de sentimientos que inicialmente no se esperaba experimentar. En este marco, pudieron identificarse dos procesos diferenciados que incidieron en el modo de comprender y vivir el propio rol de extensionista. Por un lado, lo más frecuente es dejar de considerar a la labor de extensión como un simple trabajo para pasar a vivirlo como una suerte de compromiso, lo que va de la mano con experimentar satisfacción cuando los productores obtienen resultados y mejoran sus condiciones de vida: "tengo una foto que llega un niño con unos rábanos [...] esta pobre gente es feliz con un rábano, entonces a mí me gusta hacer feliz a la gente de ahí, entonces le pongo más amor a esto, más ganas [...] entonces yo miro ahí la extensión como algo que uno siente en las venas" (H.GT.46), "cuando tú ves que en medio de tu trabajo formas personas que te lo agradecen, que tienen resultados, que mejoran sus vidas, te sientes como algo tuyo" (M.CU.31).

En contrapartida, también hay extensionistas que, a partir del surgimiento de sentimientos como tristeza o enojo, plantean la necesidad de tomar distancia emocional para no sufrir, a partir de considerar a la extensión como un simple trabajo "antes me comía la cabeza tratando de que [los productores] pudieran avanzar. Hoy trato de separar. Antes me involucraba demasiado con la gente [...] porque donde me empiezo a involucrar... [...] empiezo a sufrir con ellos" (M.UY.64), "en mis primeros años llegaba con la pena en la casa, y traspasaba eso a mi casa [...] entonces llegó un momento que [...] tú dices 'este es un trabajo $[\ldots .$.$] y yo tengo un horario y tienen que respetar mi privacidad"' (H.CH.82).$ 


\section{DISCUSIÓN}

\subsection{Análisis de resultados}

El estudio realizado permitió evidenciar que una amplia mayoría de los extensionistas rurales y asesores técnicos modifican la forma de enfocar su trabajo y de comprender su rol a lo largo del tiempo en su trayectoria profesional, fundamentalmente como resultado de la práctica y la experiencia. Este resultado discute posturas que tienden a describir estos enfoques de manera cristalizada, desconociendo que éstos se van transformando a lo largo del tiempo. En contraste, los resultados apoyan la afirmación de que se requiere tiempo para generar cambios significativos en los enfoques y en los modos en que los extensionistas comprenden su rol (Minh et al., 2010; Chowdhury et al., 2014). Como se señaló en la introducción, no pueden esperarse cambios significativos a partir de intervenciones puntuales.

Como parte de la investigación, se identificaron tres fuentes posibles de cambios en los enfoques de los extensionistas y en la comprensión de su rol: nuevas demandas o desafíos del contexto, cambios institucionales, y la práctica y la experiencia. No obstante, el análisis también contribuyó a diferenciar entre cambios en el modo de hacer extensión que se dan como resultado de restricciones externas o transformaciones en los entornos (por ejemplo, falta de vehículo para trasladarse o la institucionalización de un nuevo programa que exige nuevas prácticas), y cambios que expresan un convencimiento personal e interno de los propios extensionistas.

Los resultados muestran que cambios en el contexto y en las propuestas institucionales pueden influir en el modo subjetivo de pensar la extensión que tienen los extensionistas tanto a partir de estrategias formativas (talleres y capacitaciones) como de generar nuevos espacios de práctica y experiencia. En general, la bibliografía académica parece hacer focalizado en la capacidad transformadora de capacitaciones o talleres en los enfoques de los extensionistas (e.g. Landini \& Brites, 2018; Nettle et al., 2018), quedando invisibilizado el rol del contexto y de las instituciones en la apertura de nuevos campos de experiencia o escenarios de práctica. Por ejemplo, la decisión del MAGA en Guatemala de trabajar con amplios sectores de población en situación de desnutrición indudablemente va a generar un impacto en el modo en que los extensionistas viven afectivamente su propio rol y se relacionan con su trabajo. De esto se sigue que las instituciones de extensión pueden impulsar ciertos aprendizajes y ciertos cambios en los enfoques de sus extensionistas a partir de facilitar o limitar ciertas experiencias. Incluso, dentro de este esquema, podría pensarse que las capacitaciones o talleres formativos pueden ocupar el rol de espacios catalizadores de cambios que se basan en la consolidación de la experiencia de los participantes y se apoyan en el intercambio entre pares (véase Landini et al., 2017)

Se identificaron una serie de cambios frecuentes en los enfoques de extensión y en las formas de comprender el rol de extensionistas entre los entrevistados. El primero mencionado fue el pasaje de una visión de la extensión limitada a aspectos y procesos exclusivamente técnico-productivos, a una perspectiva más amplia que incluye otras áreas de interés como lo comercial, lo organizativo y lo agroindustrial, que visibiliza la dimensión pedagógica del rol extensionista y que reconoce la necesidad de incluir variables sociales y familiares en los diagnósticos. Este resultado va en línea con diferentes autores que plantean que los profesionales de las ciencias agropecuarias suelen tener una visión reducida de los agroecosistemas, lo que limita su comprensión de relaciones sistemáticas complejas (Salomonsson et al., 2009; Sarandón \& Flores, 2014), y con aquellos trabajos que destacan la amplitud, complejidad y diversidad del rol de extensionista (Sulaiman \& Davis, 2012). Al mismo tiempo, este resultado pone en cuestión el amplio predominio de extensionistas con formación en ciencias agropecuarias y la escasa presencia de profesionales formados en ciencias sociales (Landini \& Bianqui, 2014), y hace cada vez más evidente la necesidad de que el trabajo de extensión sea llevado adelante por equipos interdisciplinarios (Catullo et al., 2014).

El segundo eje corresponde a un cambio en el enfoque de extensión propiamente dicho, desde una visión difusionista, orientada a la transferencia de tecnologías, a una 
caracterizada por procesos de diálogo y negociación donde se reconoce el papel central que juegan los objetivos y prioridades de los productores. Este resultado es consistente con el trabajo realizado por Landini et al. (2013) en Paraguay, quienes concluyeron que la orientación difusionista de los extensionistas disminuye con la edad y la experiencia, y con las capacitaciones descriptas por Landini \& Brites (2018) y por Nettle et al. (2018), quienes identificaron cambios similares como resultado de procesos formativos. En términos conceptuales, este cambio también coincide con las transformaciones históricas acontecidas en los modelos de extensión e innovación rural a nivel internacional (Leeuwis \& Aarts, 2011), y con las críticas recibidas en América Latina por los enfoques lineales o difusionistas, todo lo que ha llevado al reconocimiento de la necesidad de implementar metodologías de extensión constructivistas centradas en el diálogo, la facilitación y la construcción de consensos (Machado et al., 2006).

Este estudio también muestra que el cambio a nivel subjetivo de un enfoque a otro se apoya en la experiencia de que los productores no aceptan de manera directa o irreflexiva lo que se les propone, lo que hace visible a los extensionistas que los productores poseen una perspectiva o una forma de ver los problemas diferente a la que tienen ellos. A partir de esta investigación se reconoce que es fundamental que el vínculo de extensión sea horizontal, entendido como una relación donde no existen jerarquías, pero que no sea percibido como un vínculo entre iguales, ya que esto invita a invisibilizar las diferencias que existen entre ambos. En efecto, los resultados invitan a pensar el vínculo entre extensionistas y productores como un uno entre 'diferentes', es decir, como un vínculo que se encuentra organizado y estructurado a partir del hecho de que sus participantes poseen conocimientos, valores y objetivos diferentes. De esta forma, el concepto de interfaz social, entendido como espacio donde se articulan actores con diferentes conocimientos, intereses y poder (Long, 2007) aparece como particularmente propicio para pensar el vínculo técnico-productor (Landini, 2016a).

Otro de los cambios identificados en este estudio en los enfoques de los extensionistas como consecuencia de la práctica y la experiencia es un mayor realismo relativo a lo que puede o no ser alcanzado con el trabajo de extensión y la pérdida de la visión idealizada que algunos traían de los productores. En general, este resultado no parece haber sido reportado por otros autores. En particular, esto evidencia la importancia que tiene moderar las expectativas de los extensionistas en momentos iniciales de práctica, tanto en instancias de formación como en espacios de socialización institucional.

Diferentes procesos analizados vinculados con cambios en los enfoques de extensión se encuentran relacionados con las identidades de los extensionistas. En primer lugar, se observa que el reconocimiento de que la extensión rural es una práctica más amplia y compleja que lo que inicialmente se preveía, pone en cuestión la identidad de 'profesionales técnicos' de muchos extensionistas. Nettle et al. (2018) señalan que plantearse un nuevo espacio de acción genera incertidumbre subjetiva y percepción de riesgo. En este caso, es posible prever cierta tensión entre la aceptación del nuevo rol (con la consiguiente incertidumbre asociada), y la permanencia dentro de un modelo de práctica técnica, conocido pero limitado para resolver los desafíos que propone el trabajo cotidiano.

Por su parte, la toma de conciencia de las limitaciones del propio conocimiento, junto con el reconocimiento del valor de los saberes empíricos de los productores, también tiene implicancias identitarias. A nivel de autoestima, implica poner en cuestión una fuente de orgullo personal y profesional, como son los conocimientos y títulos obtenidos en institutos de formación y universidades. Y a nivel propiamente identitario, implica abandonar el rol de expertos para adoptar uno más cercano al de facilitador, sin que se perciba contar con suficientes herramientas para sumirlo plenamente, como observaron Cerf et al. (2011).

Por último, también se observó que el contacto con personas en situación de pobreza, incluso indigencia, llevó a que varios extensionistas se sintieran agradecidos por lo que tenían, una autopercepción surgida a partir de poder compararse con otros, un proceso que ha sido conceptualizado en términos de 'identidades contrastivas' (Landini, 2014). Así, puede concluirse que los cambios en los enfoques de extensión y en la comprensión del rol como extensionista se encuentran articulados de manera constitutiva con la autoestima y la 
identidad, lo que implica reconocer que cambios a este nivel usualmente impactarán no solo en los extensionistas como profesionales sino también como personas.

Un segundo argumento que refuerza la afirmación precedente es el reconocimiento de la dimensión emocional relacionada con la práctica de extensión y, en particular, con el cambio en los enfoques y la comprensión del rol de extensionista. En un primer sentido, los resultados evidenciaron que el trabajo de extensión puede llevar a sentir de manera cercana la situación de necesidad de productores que viven situaciones difíciles, a construir relaciones de afecto con los agricultores, a sentir enojo y frustración a causa de proyectos que no se concretan, y a sentir satisfacción cuando las personas salen adelante como resultado del apoyo recibido. No obstante, las reflexiones en torno al impacto de la práctica y la experiencia en las identidades de los extensionistas mostraron que las emociones también están implicadas en otros procesos analizados. En efecto, la ampliación y complejización del rol del extensionista tiende a generar incertidumbre, mientras que la pérdida del rol de experto cuestiona una fuente clave de autoestima. Incluso, es claro que tanto un mayor realismo como la desidealización de los productores van a tender a minar sentimientos de entusiasmo.

Tanto Cerf et al. (2011) como Nettle et al. (2018) sugirieron la existencia de un vínculo entre cambios en las prácticas, transformaciones en las identidades y surgimiento de sentimientos y afectos. En este estudio se observó que el cambio en los enfoques de extensión y en la comprensión del rol como extensionistas se encuentra atravesada profundamente por el surgimiento de afectos y emociones, lo que no debe ser invisibilizado. Esto sugiere que la capacidad para reflexionar y manejar estas emociones es un factor clave para pasar de un enfoque de difusión lineal de tecnologías a otro más cercano a procesos de facilitación apoyados en el diálogo.

\subsection{Limitaciones y futuras líneas de investigación}

En este artículo se estudió de qué manera cambian los enfoques de extensión y la comprensión del rol de extensionista a partir de la práctica y la experiencia. De esta forma, se describió y analizó un conjunto de cambios frecuentes, lo que amplió nuestro conocimiento de la temática. No obstante, debe tenerse presente que no se trata de cambios que se den ni en todos los extensionistas o por igual en todos los países. Al mismo tiempo, la investigación tampoco relacionó cambios específicos con cantidad de años de experiencia en el rol de extensionista o con momentos puntuales de la trayectoria profesional. Por ejemplo, es posible que algunos cambios en los enfoques de extensión sean más frecuentes en los años iniciales de práctica, mientras que otros requieran mucha más experiencia en el cargo.

Nettle et al. (2018) han señalado que el vínculo con pares y la retroalimentación en el trabajo con productores constituyen un soporte sumamente valioso para impulsar cambios en las identidades y en el tipo de asesoramiento que ofrecen extensionistas y asesores. En paralelo, Landini et al. (2017) han destacado el rol central de los espacios de intercambio con otros extensionistas como motores de procesos de aprendizaje vinculados con la práctica. En este trabajo se buscó comprender cambios en los enfoques de extensión apoyados en la práctica y la experiencia de los extensionistas. Sin embargo, no se profundizó ni en los procesos cognitivos ni en las dinámicas de intercambio entre pares que acompañan y dan forma a dichos cambios.

Futuros estudios podrían aportar a una comprensión más profunda de los temas abordados. En primer lugar, investigaciones de base cuantitativa podrían contribuir a relacionar cambios específicos con momentos de la trayectoria profesional o con ciertas profesiones, a valorar en términos porcentuales la incidencia de los cambios identificados y a ponderar diferentes niveles de intensidad de dichos cambios. Por su parte, nuevos estudios cualitativos podrían ayudar a comprender mejor el proceso de aprendizaje y de cambio de enfoques de extensión con base en la práctica y la experiencia, identificando mejor el rol que juegan los propios pares, los productores, y los procesos reflexivos. Por último, los resultados alcanzados también invitan a profundizar en el estudio del rol de 
la dimensión afectiva y de los procesos identitarios en la adopción de determinados enfoques de extensión.

\section{CONCLUSIONES}

El presente trabajo se propuso describir de qué manera se va transformando, a partir de la práctica y la experiencia personal, el modo en que los extensionistas rurales comprenden el trabajo de extensión y su propio rol como extensionistas o asesores. A partir de un estudio cualitativo en el que se entrevistó a extensionistas de diferentes países latinoamericanos se concluyó que los cambios más frecuentes refieren a:

(1) Ampliación y complejización de lo que se considera el campo de acción de un extensionista, pasando de un enfoque centrado en lo técnico-productivo a uno que amplía las áreas de interés y reconoce la necesidad de valorar dinámicas familiares y sociales al momento de diagnosticar procesos y dar recomendaciones.

(2) Pasaje de un modelo de difusión tecnológica centrado en la perspectiva del extensionista a uno apoyado en la horizontalidad, el diálogo y la negociación de perspectivas. Este cambio se apoya en la toma de conciencia de las limitaciones del conocimiento propio para resolver los desafíos de la práctica y en el reconocimiento de que los productores tienen objetivos, prioridades y valores diferentes a los de los extensionistas.

(3) Mayor realismo respecto de los cambios que se pueden alcanzar por medio del trabajo de extensión y desidealización del productor como persona siempre comprometida, que busca incesantemente la mejora de las condiciones de vida de su familia, de sus pares y de su comunidad.

(4) Construcción de un vínculo emocional con el trabajo de extensión, lo que puede llevar tanto a tomar distancia afectiva del trabajo (para evitar sentimientos de malestar, tristeza o angustia) como a comprometerse con el trabajo al punto de vivirlo como un proyecto personal que excede lo laboral.

En paralelo, el análisis de los resultados permitió generar diferentes reflexiones. En primer lugar, las instituciones de extensión pueden influir en el modo subjetivo en que los extensionistas comprenden su trabajo a partir de facilitar o limitar espacios de práctica y metodologías de trabajo, y de generar espacios de formación para extensionistas donde puedan compartirse experiencias y reflexionar sobre ellas. En segundo lugar, debe reconocerse que el modo en que los extensionistas comprenden su rol se encuentra profundamente articulado con dinámicas identitarias y emocionales, por lo que no puede entenderse únicamente a nivel cognitivo. En este contexto, la capacidad reflexiva y emocional de los extensionistas se reconoce como un elemento fundamental para permitir el pasaje de modelos de extensión rural difusionistas hacia propuestas centradas en el diálogo y la facilitación de aprendizajes. Finalmente, se reconoce la importancia de pensar la relación entre extensionistas y productores en términos horizontales, pero siempre teniendo presente que ambos son diferentes y poseen visiones de mundo diferentes.

\section{REFERENCIAS BIBLIOGRÁFICAS}

Aguirre, F. (2012). El nuevo impulso de la extensión rural en América Latina: situación actual y perspectivas. Santiago de Chile: Red Latinoamericana de Servicios de Extensión Rural.

Albaladejo, C., Couix, N., \& Barthe, L. (2007). Learning in agriculture: rural development agents in France caught between a job identity and a professional identity. Journal of Agricultural Education and Extension, 13(2), 95-106.

Alemany, C., \& Sevilla Guzmán, E. (2007). ¿Vuelve la extensión rural? Reflexiones y propuestas agroecológicas vinculadas con el retorno y fortalecimiento de la extensión rural en América Latina. Realidad Económica, 227, 52-74.

Carrapizo, V., Giordano, G., Sanchez, G., Paredes, M., Bodrero, M., Brieva, S., \& Juarez, P. (2018). Tecnología y sociedad: análisis de procesos de innovación y cambio tecnológico en diversos territorios rurales de Argentina. Buenos Aires: INTA. 
Catullo, J., Torres, G., \& Mazzola, C. (2014). El rol de los equipos interdisciplinarios de extensión frente a los nuevos desafíos de la ruralidad en Latinoamérica. Revista Científica de Desarrollo y Extensión Rural, 2, 36-40.

Cerf, M., Guillot, M., \& Olry, P. (2011). Acting as a change agent in supporting sustainable agriculture: How to cope with new professional situations? Journal of Agricultural Education and Extension, 17(1), 7-19.

Chowdhury, A., Odame, H., \& Leeuwis, C. (2014). Transforming the roles of a public extension agency to strengthen innovation: Lessons from the National Agricultural Extension Project in Bangladesh. Journal of Agricultural Education and Extension, 20(1), 7-25.

Christoplos, I. (2010). Mobilizing the potential of rural and agricultural extension. Roma: FAO.

Da Ros, C. (2012). A contribuição das visitas de campo no ensino das ciências agrárias na UFRRJ. Revista Ciência em Extensão, 8(1), 107-122.

Díaz-Bravo, L., Torruco-García, U., Martínez-Hernández, M., \& Varela-Ruiz, M. (2013). La entrevista, recurso flexible y dinámico. Investigación en Educación Médica, 2(7), 162-167.

Diesel, V., Froehlich, J., Neumann, P., \& Silveira, P. (2008). Privatização dos serviços de extensão rural: Uma discussão (des)necessária? Revista de Economia e Sociologia Rural, 46(4), 1155-1188.

Freire, P. (1973). ¿Extensión o comunicación? La concientización en el medio rural. Buenos Aires: Siglo XXI.

Gumucio-Dagron, A. (2011). Comunicación para el cambio social: clave del desarrollo participativo. Signo y Pensamiento, 30(58), 26-39.

Höckert, J., \& Ljung, M. (2013). Advisory encounters towards a sustainable farm development: interaction between systems and shared lifeworlds. Journal of Agricultural Education and Extension, 19(3), 291-309.

Houchens, G., Hurt, J., Stobaugh, R., \& Keedy, J. (2012). Double-loop learning: a coaching protocol for enhancing principal instructional leadership. Qualitative Research in Education, 1(2), 135-178.

Ingram, J. (2008). Agronomist-farmer knowledge encounters: an analysis of knowledge exchange in the context of best management practices in England. Agriculture and Human Values, 25(3), 405-418.

Klerkx, L., Van Mierlo, B., \& Leeuwis, C. (2012). Evolution of systems approaches to agricultural innovation: concepts, analysis and interventions. In I. Darnhofer, D. Gibbon \& B. Dedieu (Eds.), Farming systems research into the 21st century: the new dynamic (pp. 457-483). Dordrecht: Springer.

Landini, F. (2013). Problemas enfrentados por los extensionistas rurales argentinos en el ejercicio de su labor desde su propia perspectiva. Revista de Economia e Sociologia Rural, 51(Supl.1), s079-s100.

Landini, F. (2014). Reflexiones teóricas y metodológicas en torno al estudio y conceptualización de la identidad. Cuadernos de la Facultad de Humanidades y Ciencias Sociales, 46, 119-135.

Landini, F. (2016a). Unfolding the knowledge and power dynamics of the 'farmers-rural extensionists' interface in North-Eastern Argentina. Journal of Agricultural Education and Extension, 22(5), 399-413.

Landini, F. (2016b). Concepción de extensión rural en 10 países latinoamericanos. Andamios, 13(30), 211-236.

Landini, F., \& Bianqui, V. (2014). Socio-demographic profile of different samples of Latin American rural extensionists. Ciência Rural, 44(3), 575-581.

Landini, F., Bianqui, V., \& Crespi, M. (2013). Evaluación de las creencias sobre extensión rural de los extensionistas paraguayos. Psiencia, 5(1), 3-14.

Landini, F., \& Brites, W. (2018). Evaluation and impact of a reflective training process for rural extension agents. Journal of Agricultural Education and Extension, 24(5), 457-472.

Landini, F., Brites, W., \& Mathot, M. (2017). Towards a new paradigm for rural extensionists' in-service training. Journal of Rural Studies, 51, 158-167.

Leeuwis, C. (2004). Communication for rural innovation: rethinking agricultural extension. Oxford: Blackwell Science.

Leeuwis, C., \& Aarts, N. (2011). Rethinking communication in innovation processes: creating space for change in complex systems. Journal of Agricultural Education and Extension, 17(1), 21-36.

Long, N. (2007). Sociología del desarrollo: una perspectiva centrada en el actor. México: CIESAS.

Machado, J., De Hegedüs, P., \& Silveira, L. (2006). Estilos de relacionamento entre extensionistas e produtores. Ciência Rural, 36(2), 641-647. 
Minh, T., Larsen, C., \& Neef, A. (2010). Challenges to institutionalizing participatory extension: The case of farmer livestock schools in Vietnam. Journal of Agricultural Education and Extension, 16(2), 179194.

Mojarradi, G., \& Karamidehkordi, E. (2016). Factors influencing practical training quality in Iranian agricultural higher education. Journal of Higher Education Policy and Management, 38(2), 183-195.

Moschitz, H., Roep, D., Brunori, G., \& Tisenkopfs, T. (2015). Learning and innovation networks for sustainable agriculture: Processes of co-evolution, joint reflection and facilitation. Journal of Agricultural Education and Extension, 21(1), 1-11.

Movahedi, R., \& Nagel, U. (2012). Identifying required competencies for the agricultural extension and education undergraduates. Journal of Agricultural Science and Technology, 14(4), 727-742.

Nettle, R., Crawford, A., \& Brightling, P. (2018). How private-sector farm advisors change their practices: an Australian case study. Journal of Rural Studies, 58, 20-27.

Otero, J., \& Selis, D. (2016). La revista "Extensión en las Américas". Influencia de los EEUU en los servicios de extensión rural latinoamericanos. Extensão Rural, 23(1), 42-57.

Rivera, W. (2011). Public sector agricultural extension system reform and the challenges ahead. Journal of Agricultural Education and Extension, 17(2), 165-180.

Salomonsson, L., Nilsson, A., Palmer, S., Roigart, A., \& Francis, C. (2009). Farming systems education: Case study of Swedish test pilots. Renewable Agriculture and Food Systems, 24(1), 48-59.

Sarandón, S., \& Flores, C. (2014). Agroecología: bases teóricas para el diseño y manejo de agroecosistemas sustentables. La Plata, Argentina: Universidad Nacional de La Plata.

Shukri Al-Rimawi, A., Allahyari, M., \& Al-Rusheidat, J. (2017). Assessing extension agent training needs, barriers and training methods in Jordan. Journal of Agricultural Science and Technology, 19(5), 1019 1029.

Sulaiman, R., \& Davis, K. (2012). El "Nuevo Extensionismo": funciones, estrategias y capacidades para reducir el hambre y la pobreza. Lindau, Suiza: Global Forum for Rural Advisory Services.

Tarekegne, C., Wesselink, R., Biemans, H., \& Mulder, M. (2017). Developing and validating a competence profile for development agents: an Ethiopian case study. Journal of Agricultural Education and Extension, 23(5), 427-441.

Taylor, S., \& Bogdan, R. (1990). Introducción a los métodos cualitativos de investigación. Buenos Aires: Paidos.

Thornton, R. (2006). Los 90 y el nuevo siglo en los sistemas de extensión rural y transferencia de tecnología públicos en el MERCOSUR. Santa Rosa, Argentina: INTA.

Torrado Porto, R., \& Catullo, J. (2017). Extensión rural y enfoque territorial: aprendiendo en la acción con otros. Revista de la Facultad de Agronomía, 116, 19-27.

Zuin, L., Zuin, P., \& Manrique, M. (2011). A comunicaçâo dialógíca como fator determinante para os processos de ensino aprendizagem que ocorrem na capadtaçâo rural: Um estudo de caso em um órgao público de extensäo localizado no interior do Estado de São Paulo. Ciência Rural, 41(5), 917923. 